\title{
Fetal Hemodynamic Profile in Splanchnic Vessels Centralization Mechanism Analysis
}

\author{
JM Troyano, M Alvarez de la Rosa, A Padilla, L Ces, LT Merce \\ Division of Ultrasound and Fetal Medicine, Department of Obstetrics and Gynecology, University Hospital of the Canary Islands \\ Tenerife, Spain \\ Correspondence: JM Troyano, Professor of Obstetrics and Gynecology, University of La Laguna, Tenerife, Spain \\ e-mail: Jtrayanol@sego.es
}

\begin{abstract}
We compared the hemodynamic profiles of abdominal visceral vessels in physiological and pathological conditions as well as peripheral vessels away from the visceral area.

The resistance index (RI) was monitored in the renal, celiac trunk, gastric, pancreatic and spleen artery linearly in a bi-monthly bases, in 159 physiological pregnancies.

On the other hand an additional group of 47 pregnancies between the 26th and the 40th weeks of gestation were monitored for differences in RI from the renal artery in both right and left kidney. The RI from the right kidney was always lower than in the left kidney, a difference that was nonetheless not statistically significant.

Concerning hemodynamic responses to fetal pathology, kidney hemodynamics were surveyed in two samples of fetuses displaying IUGR. The first sample was made up of a total of 72 fetuses showing IUGR associated with euamnion, while the second sample consisted on 39 IUGR fetuses showing oligohydramnion.

The renal artery in IUGR-euamnion fetuses yielded RI values similar to those recorded from healthy kidneys.

In contrast, in IUGR-oligohydramnion cases, the RI was markedly restrictive when compared to that of healthy and IUGR-euamnion situations.

Besides we investigated in 49 cases the hemodynamic behavior in macrosomic fetuses in diabetes pregnancy, between 32nd to 38th week.

Vascular resistance affecting blood flows in fetal kidneys is lower in macrosomic fetuses with gestational diabetes than in normal gestations with statistical significance.

In cases of kidney parenchyma pathology 49 pregnancies presented severe hydronephrosis, 37 pregnancies had light hydronephrosis and 22 pregnancies were diagnosed as cases of corticomedullar dysplasia associated with oligohydramnion.

RI values from the renal artery were higher in cases of severe hydronephrosis than in cases of light hydronephrosis. This difference was statistically significant.

The RI obtained from cases with mild hydronephrosis was fairly similar to that of healthy kidneys,

In contrast, the RI values recorded from fetuses having an association of corticomedullar dysplasia and oligohydramnion were the highest among the three pathological groups.

Regarding splanchnic and other abdominal viscera, celiac trunk's spectral profile were characterized by a low resistance, and showed a gradual increase in diastolic velocity throughout gestation.
\end{abstract}

The gastric artery displayed a gradually declining vascular resistance through pregnancy.

Hemodynamic records from the pancreatic artery are restrictive over the whole period of gestation and only the maximum systolic peak can be subjected to measurement.

The spleen artery showed a low diastolic resistance which declined markedly through gestation, even in situations of chronic fetal compromise, this artery experienced increased vascularization in response to stress, a vascular trait that was found to be unique for this anatomical area.

The flowmetric resistance in the spleen artery was lower than in the renal artery and than the rest of the abdominal vessels studied.

The femoral artery had a very restrictive hemodynamic profile, which was characterized by the lack of a telediastolic flow.

These data suggests that the fetal blood stores are located in the extremities rather than in visceral areas.

The monitoring of the hemodynamic patterns emerging from the vessel complex studied here, has allowed an insight into the mechanism of vascular centralization in response to perturbing conditions, this mechanism should be regarded as a kind of functional and nutritional autoregulative reaction of the visceral set involved.

Keywords: Hemodynamic profile, splanchnic vessels, fetal abdominal vessels, peripheral vessels, centralization mechanism.

\section{INTRODUCTION}

Given the complexity of the vascular network of the fetus, could it be asserted that, in response to stress, some fetal viscera experience important blood restrictions that favor and ensure the irrigation of essential tissues, such as myocardium, adrenal gland and brain?

The answer is yes indeed. The hemodynamic behavior of the kidneys is a representative example of this. It is known that reductions in kidney perfusion do result from situations of fetal suffering and lead to typical oligohydramnion, often associated with intrauterine growth restriction (IUGR). ${ }^{1}$

Concomitantly, an increase in cerebral vascularization takes place in the same context of stress and keeps up the activity of the brain. 2,3 
Two main functions can be attributed to the vascular system of the kidneys:

- Nourishment of the kidney parenchyma.

- Production and clearing of urine.

The complexity of the kidney vascularization patterns can be contrasted on the facts that: (1) hemodynamic responses to stimuli are fairly variable, and that (2) kidney functionality is regulated by up to five agents, namely:

- The sympathetic nervous system

- The arginine-vasopressin system

- Levels of angiotensine

- Levels of prostaglandins (PGI-2)

- Heart store

In response to chronical stress, the fetus is able to minimize water losses by diminishing its diuretic effort. This is not a subsidiary event but a prime mechanism that warrants the systemic perfusion of the fetus and, in particular, the perfusion of the kidney parenchyma in situations of blood centralization.

Overall, this mechanism involves the redistribution of blood among and in each viscera in situations of fetal compromise, and thus should not be regarded as a simple event of centralization-restriction.

In assessing renal hemodynamics, two main factors must be taken into account:

1. On one hand: Glomerullar filtration, which is dependant on a number of parameters including hydrostatic filtration, oncotic pressure, capillar permeability, and total area comprising capillar glomerullar tissue.

2. On the other hand: Total renal flow, which is dependant on cardiac blood stores, vascular tone of renal interstitia, glomerullar resistance and tubular reabsortion.

The application of the same rationale should be widened to assess hemodynamic patterns of other viscera that, under stressing conditions, also change their vascularisation in order to ensure nutritional inputs and functional performance.
There is evidence for arguing that under the effect of a given aggression, the first reaction of the fetus consists of a functional reorganisation that may guarantee an homeostatic balance for coping with any further emerging strong physiological restriction

In this report, we compared hemodynamic profiles in physiological and pathological conditions from visceral as well as peripheral vessels away from the visceral area.

Thus, various vessels were selected for monitoring as follows:

Visceral vessels: The renal artery, and celiac trunk, spleen, pancreatic, and gastric arteries, both of which are embodied in the splanchnic area.

Peripheral vessels: The femoral artery, a vessel that can be easily located by power Doppler, unlike other peripherals such as the radial artery whose localization is rather complicated.

The resistance index (RI) was monitored in all these visceral vessels, and in the renal artery linearly, in a bi-monthly bases, in 159 physiological pregnancies.

Regarding renal artery, there was a decrease in the RI from the renal artery throughout pregnancy, particularly from the 32nd gestational week onwards, a trend possibly caused by the changes accompanying the final stages of the centrifuge nephrogenesis (Fig. 1).

Moreover, a light telediastolic response was detected in association with a protodiastolic notch typically resulting from tissular resistance.

A further group of 47 pregnancies between the 26th and the 40th weeks of gestation were monitored for differences in RI from the renal artery in both the right and the left kidney (Fig. 2).

The RI from the right kidney was always lower than that in the left kidney, a difference that was nonetheless not statistically significant.
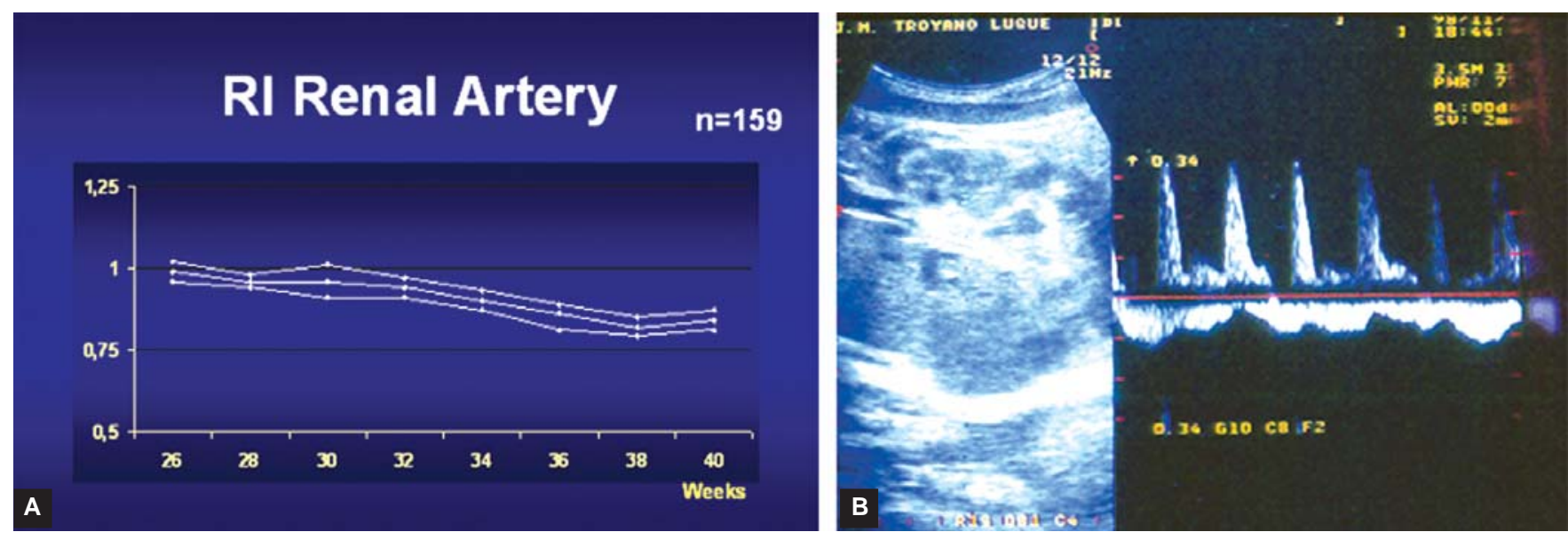

Figs $1 \mathrm{~A}$ and B: Resistance Index (RI). Slope profile in normal pregnancy and flow hemodynamic profile (artery and vein) 


\section{HEMODYNAMIC RESPONSES TO FETAL PATHOLOGY}

Concerning hemodynamic responses to fetal pathology, kidney hemodynamic were surveyed in two samples of fetuses displaying IUGR., ${ }^{4,5}$

The first sample was made up of a total of 72 fetuses showing IUGR associated with euamnion, while the second sample consisted of 39 fetuses presenting IUGR in association with oligohydramnion (Fig. 3).
The renal artery in IUGR-euamnion fetuses showed RI values similar to those recorded from healthy kidneys.

In contrast, in IUGR-oligohydramnion cases, the RI was markedly restrictive when compared to that of healthy and IUGReuamnion situations.

On the other hand, we tried to investigate in 49 cases the hemodynamic behaviour in macrosomic fetuses in diabetes pregnancy, between 32nd to 38th week (Fig. 4).

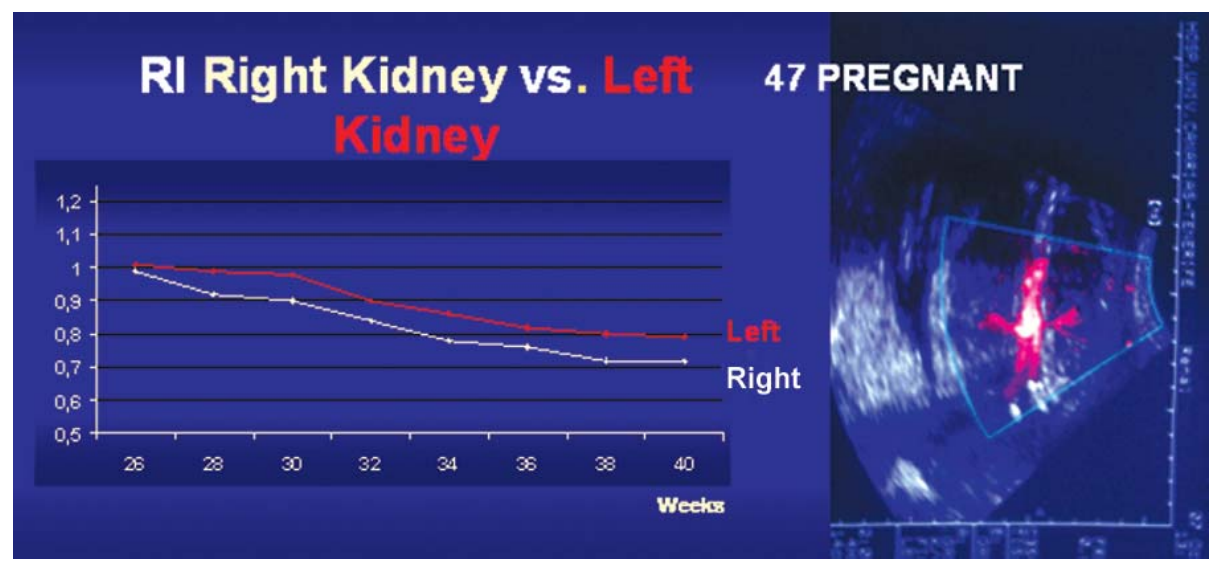

Fig. 2: Renal artery. Differences in RI from the renal artery in both the right and the left kidney
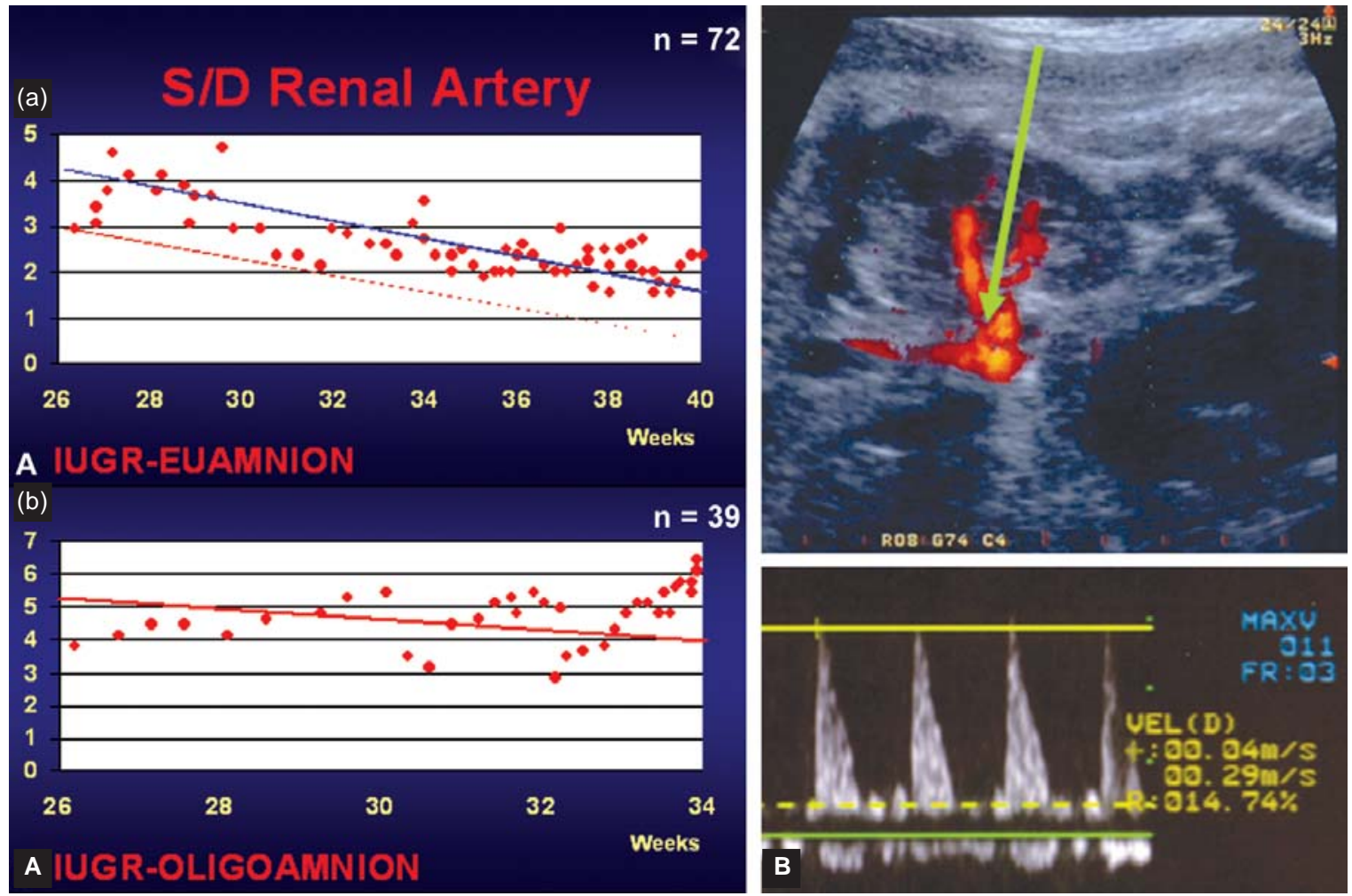

Figs 3A and B: (A) In IUGR and euamnion fetuses yield RI values similar to healthy fetuses. (B) In IUGR —oligohydramnios cases, the $\mathrm{RI}$ is markedly restrictive when compared to that of healthy and IUGR-euamnion situation 


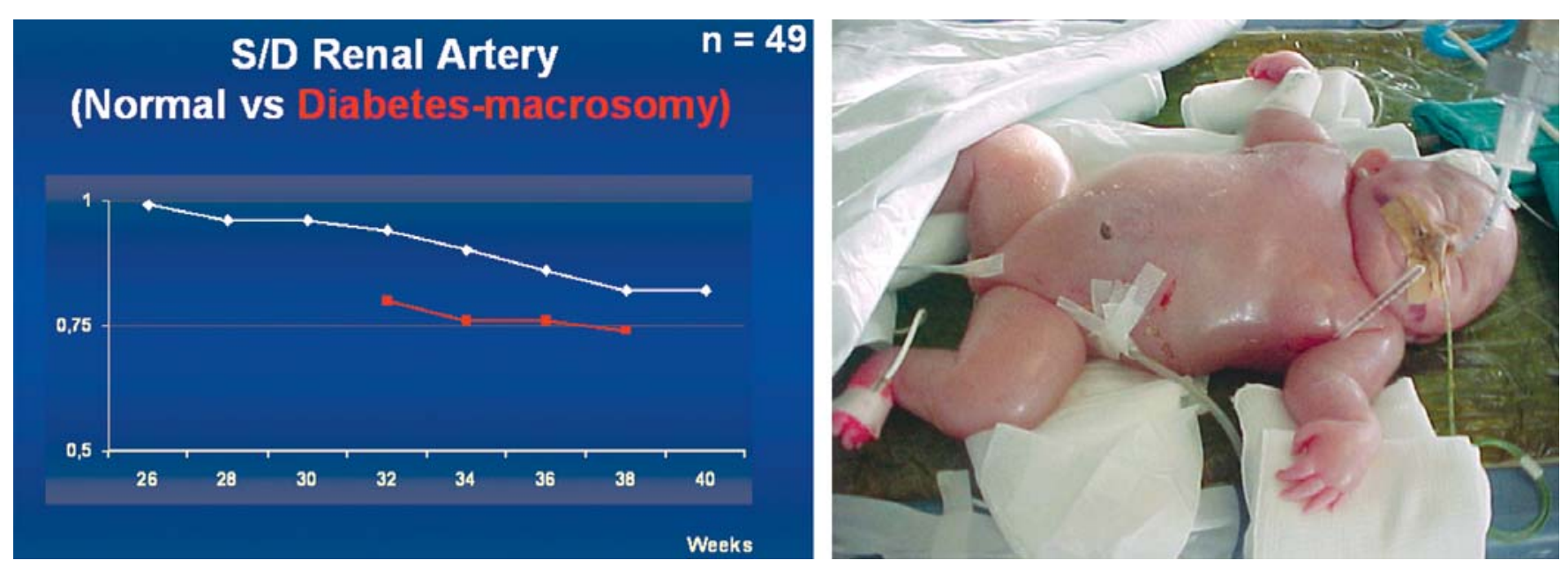

Fig. 4: Comparative profile slope between macrosomic fetuses in diabetes pregnancy vs normal conditions

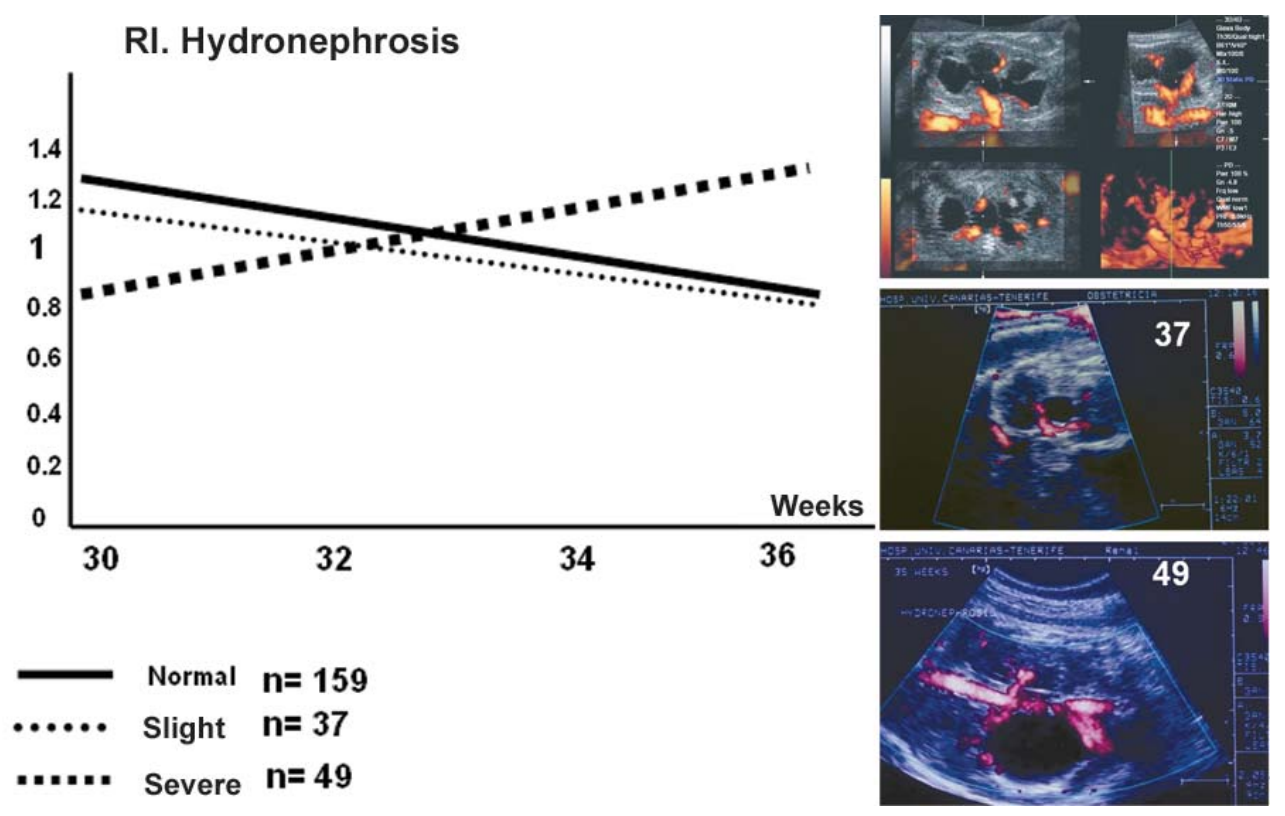

Fig. 5: RI Comparative profile slope among severe, mild hydronephrosis and normal pregnancy

Vascular resistance effecting blood flows in fetal kidneys is lower in macrosomic fetuses with gestational diabetes than in normal gestations.

\section{HEMODYNAMIC RESPONSES TO PATHOLOGY OF KIDNEY PARENCHYMA}

With regard to hemodynamic responses to pathology of renal parenchyma the RI was monitored in the renal artery of fetuses having nephrourological pathologies in both the parenchymatous tissue and excretory function.

Firstly, a total of 49 pregnancies presented severe hydronephrosis (Fig. 5).
Secondly a further 37 pregnancies had mild hydronephrosis.

A final group of 22 pregnancies were diagnosed as cases of corticomedullar dysplasia associated with oligohydramnion. (Fig. 6).

RI values from the renal artery were higher in cases of severe hydronephrosis (without and obvious parenchyma) than in cases of mild hydronephrosis. This difference was statistically significant except when the parenchyma was visible. Furthermore, the RI obtained from cases with mild hydronephrosis was fairly similar to that of healthy kidneys, possible due to the existence of patches of nephrones that kept their functionality. 

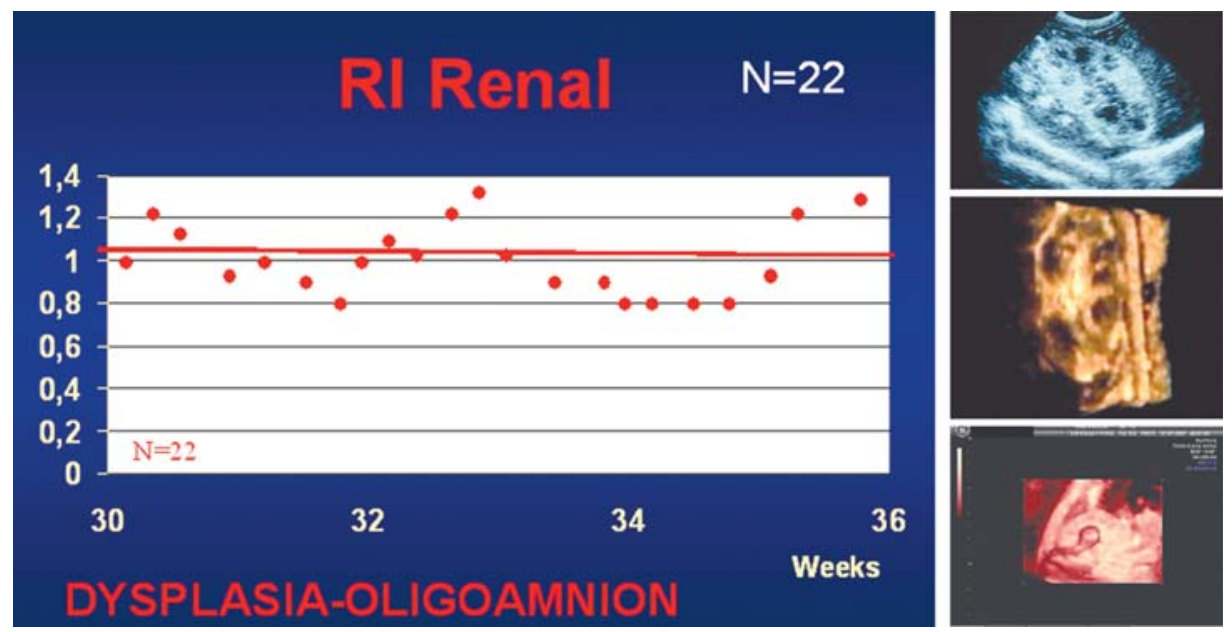

Fig. 6: RI profile slope in 22 cases of corticomedullar dysplasia associated with oligohydramnion

In contrast, the RI values recorded from fetuses having an association of corticomedullar dysplasia with oligohydramnion were the highest among the three pathological groups, showing a nearly horizontal evolutive slope.

Severe impacts on the flowmetric patterns of the renal artery resulted from lesions of the renal parenchyma, and were much greater than those exerted by obstructive pathologies unless associated with parenchymatous alterations.

On this ground, it was interesting to study the hemodynamic behaviour of different vessels running close to the kidneys. ${ }^{6}$

The superior mesenteric artery, irrigates the whole of the digestive tube and displays a very strong hemodynamic record. This vessel runs by the area where both the right and left renal arteries experience anastomosis with the aorta, and goes particularly close to the left renal artery ${ }^{6,7}$ (Fig. 7).

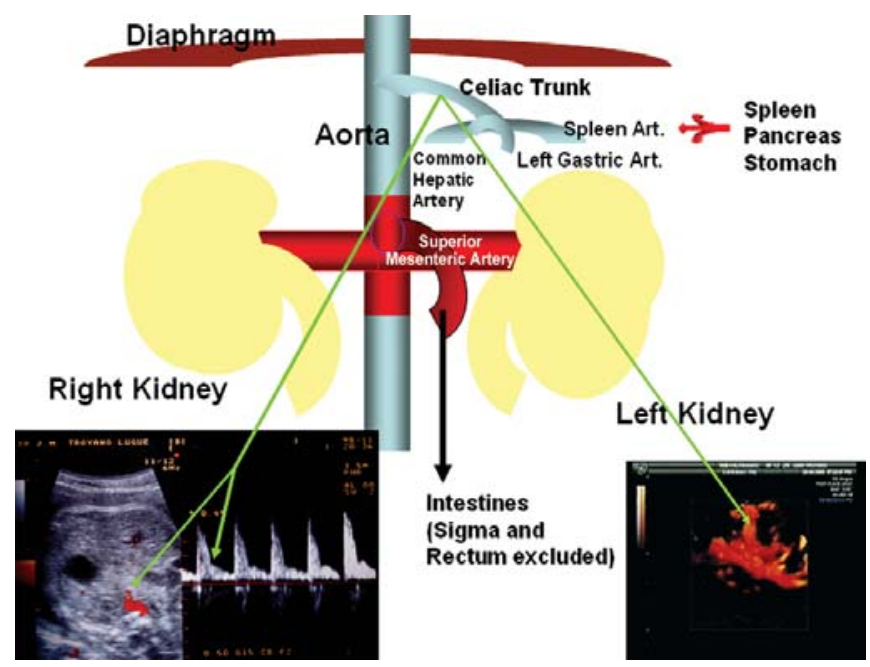

Fig. 7: Abdominal vessels scheme
Therefore, contrasting hemodynamic patterns could be expected between both renal arteries under the differential influence of the mesenteric vessel.

Just over the area of anastomosis described, at a subdiaphragmatic level, a large arterial vessel, the celiac trunk, also experiences anastomosis and extends towards a ventral direction from the aorta. Several vessels branch off from the celiac trunk and irrigate the stomach and spleen. In turn, a number of vessels depart from the spleen branch, including the gastric and pancreatic arteries and the most important vessels in terms of blood flows: The splenic artery. ${ }^{8}$

The abundant blood flow channelled through the spleen artery does give a strong hemodynamic signal that can be unmistakenly identified, however, the vascular characterisation of the remaining vessels within this area is not easy and will be only tackled as we achieve further monitoring progress in the time to come. ${ }^{8}$

Nonetheless, different aspects of the hemodynamic performance of this complex anatomical area have been assessed in physiological condition.

The celiac trunk can be detected at a subdiaphragmatic level, just in front of the aorta, forming an angle of $90^{\circ}$ with the longitudinal axis of the aortic lumen.

Spectral profiles from the celiac trunk were characterised by a low resistance, and showed a gradual increase in diastolic velocity throughout gestation (Fig. 8).

Both gastric and pancreatic vessels could be located providing that no trunkal spasms occurred and, that the activity of the stomach was in the phase of maximum gastric filling.

The gastric artery displayed an optimal hemodynamic signal with a vascular resistance declining gradually through pregnancy (Fig. 9).

The pancreatic artery is located behind the stomach and to the left of and an angle of $30^{\circ}$ with the aorta (Fig. 10). ${ }^{6}$ 

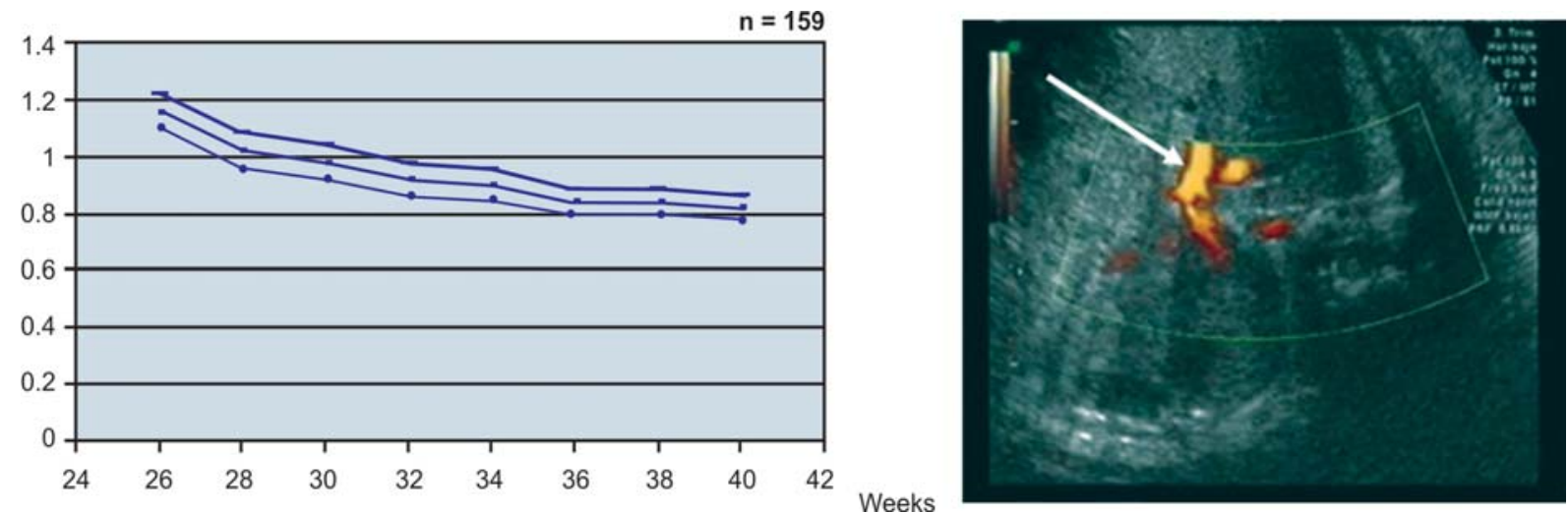

Fig. 8: Celiac trunk. Slope profile in normal pregnancy

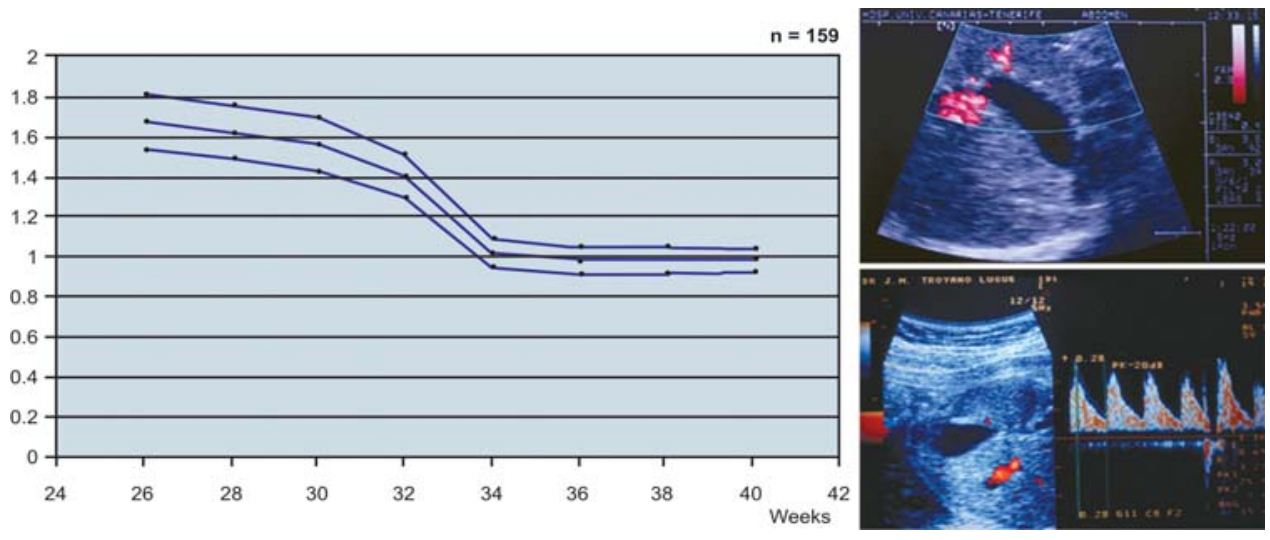

Fig. 9: Gastric artery. Slope profile in normal pregnancy

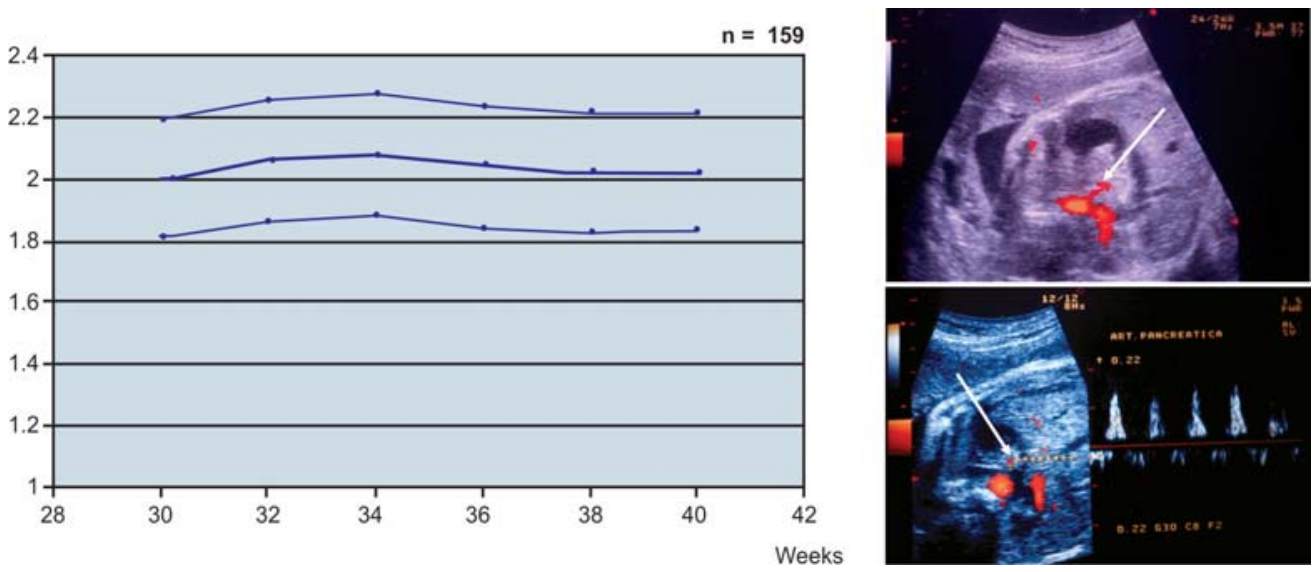

Fig. 10: Pancreatic artery. Profile slope in normal pregnancies 
Hemodynamics records from this vessel are restrictive over the whole period of gestation and only the maximum systolic peak can be subjected to measurement.

A reduction in diastolic resistance was observed in macrosomic fetuses from women presenting with gestational diabetes. It can be argued that pancreatic hyperplasia may be causing increases in visceral vascularization and could be used in the future as an indirect marker of hyperinsulinism (Fig. 4).

The spleen artery is the main vessel of this visceral area, providing a strong Doppler record besides, unlike the neighbouring vessels, the spleen artery showed a low diastolic resistance which declined markedly through gestation, even in situations of chronic fetal compromise (Fig. 11).

In fact, the spleen artery experienced increased vascularization in response to stress, a vascular trait that was unique for this anatomical area. ${ }^{8}$

Could it be concluded that the spleen's hemodynamic behaviour in reaction to hypoxic redistribution defers from that of any other viscera?

Could these changes in visceral vascularization be occurring in order to enhance the production of erythropoietin and thus counteract hypoxia?

Global phenomena such as vascular reorganization are difficult to monitor and draw an assessment on, because they involve complex multivisceral responses that keep up the complete homeostatic balance of the fetus. ${ }^{10}$

Therefore, it was interesting to survey peripheral areas having a minor role in fetal survival, and thus the femoral artery was subjected to monitoring. This vessel has the advantage that it can be easily spotted in fetal extremities by means of an AngioDoppler procedure. ${ }^{9}$

Hemodynamic patterns from the spleen artery were studied in 159 physiological pregnancies between the 28th and the 40th week of gestation, and were subsequently compared with those from the renal artery over the same gestational period (Fig. 12).
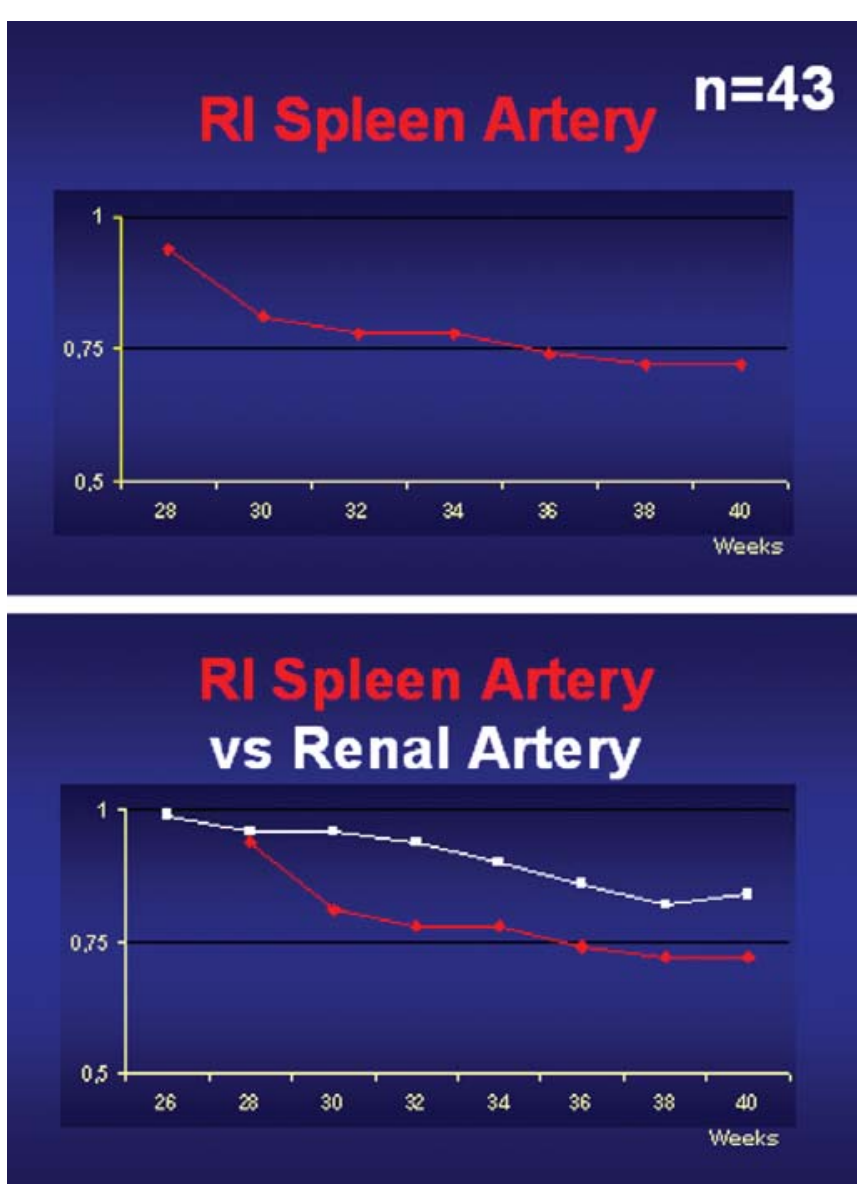

Fig. 12: Comparative slope between spleen artery vs renal artery in normal pregnancies

The flowmetric resistance in the spleen artery was lower than in the renal artery and than in any of the rest of the abdominal vessels studied.

Furthermore, the flowmetric resistance in the spleen artery declined in response to stress, while it increased in the renal artery under the same perturbing conditions (Fig. 13).
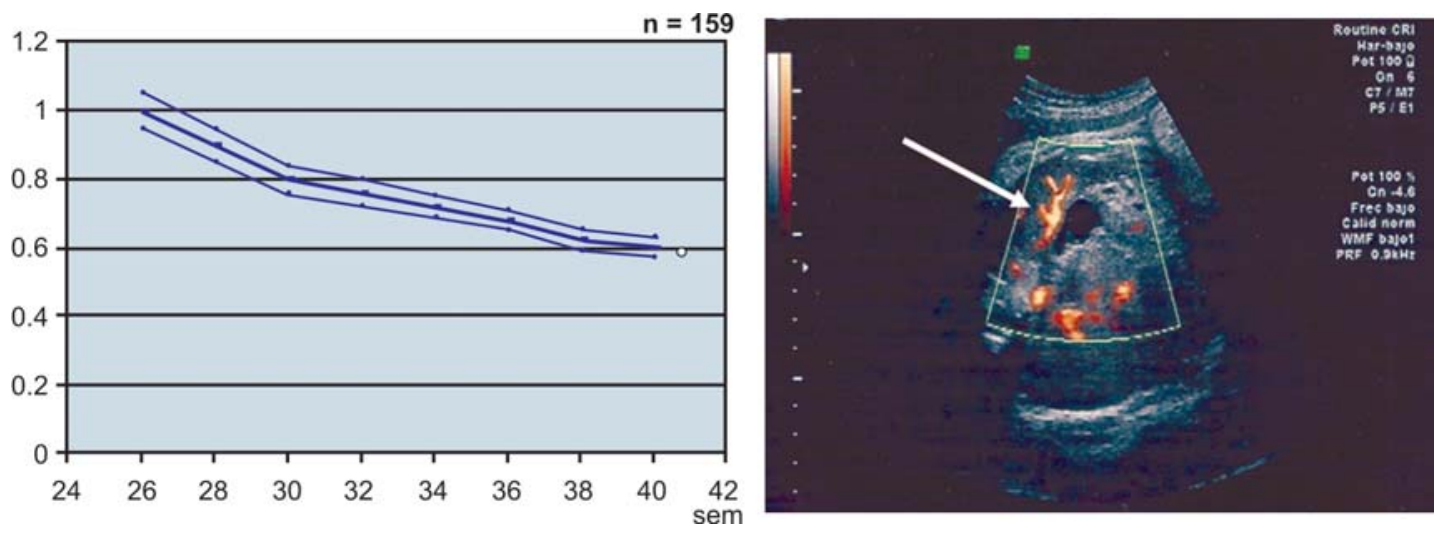

Fig. 11: Spleen artery. Profile slope in normal pregnancies 


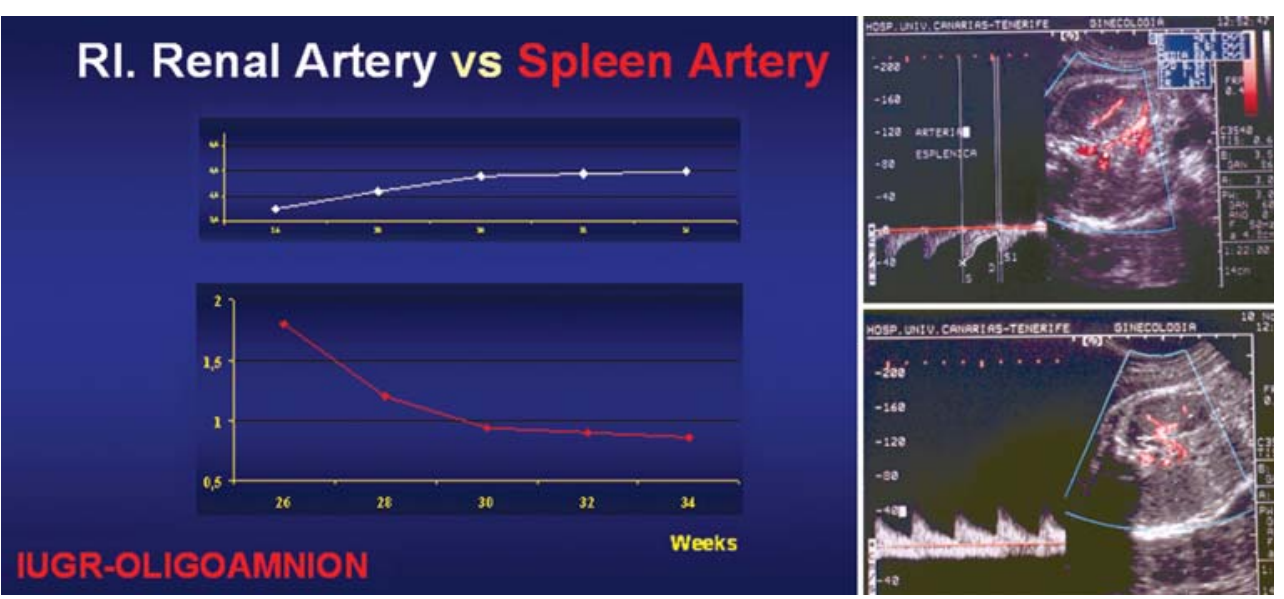

Fig. 13: Flowmetric resistance in the spleen artery declined in response to stress, while it increased in the renal artery under the same perturbing conditions

Like many other vessels (radial, poplitea, etc.), the femoral artery had a very restrictive hemodynamic profile, which was characterized by the lack of a telediastolic flow, and a systolic record increasing in the course of gestation. These data suggested that the fetal blood stores are located in the extremities rather than in visceral areas (Fig. 14).
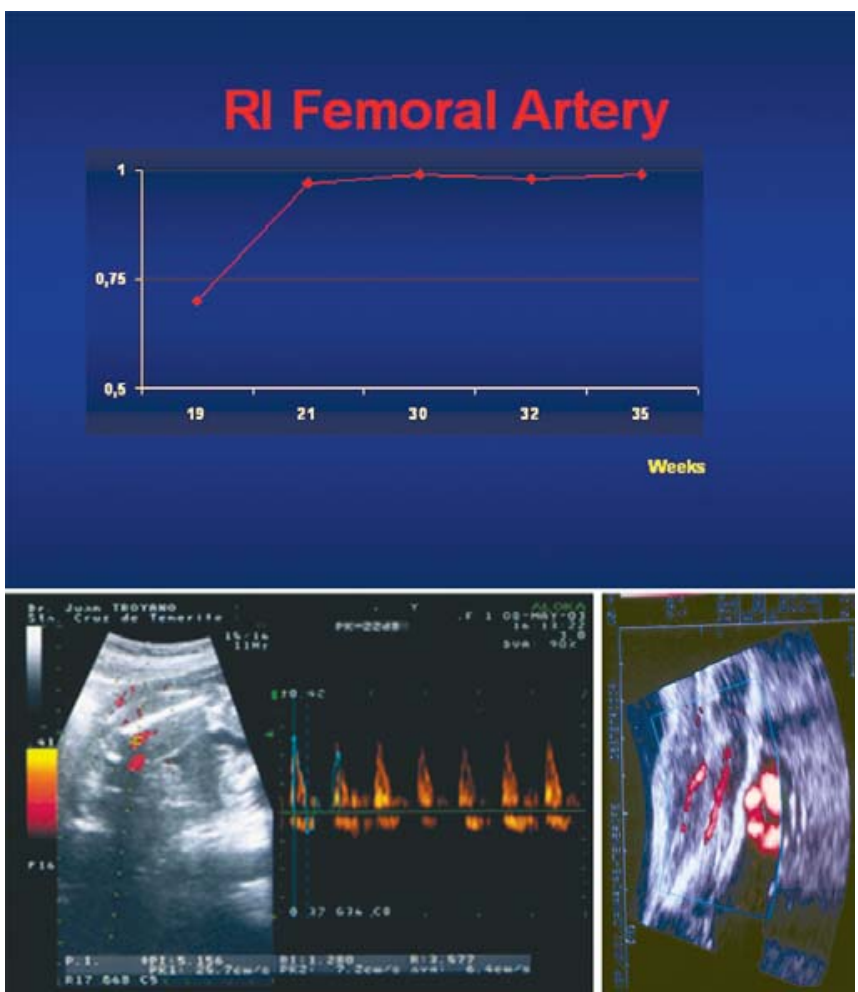

Fig. 14: The femoral artery had a very restrictive hemodynamic profile. Systolic record increasing in the course of normal gestation
A negative correlation was observed between renal and femoral flow resistances, such that renal resistance decreased as femoral resistance increased, particularly in situation of chronical fetal distress (Fig. 15).
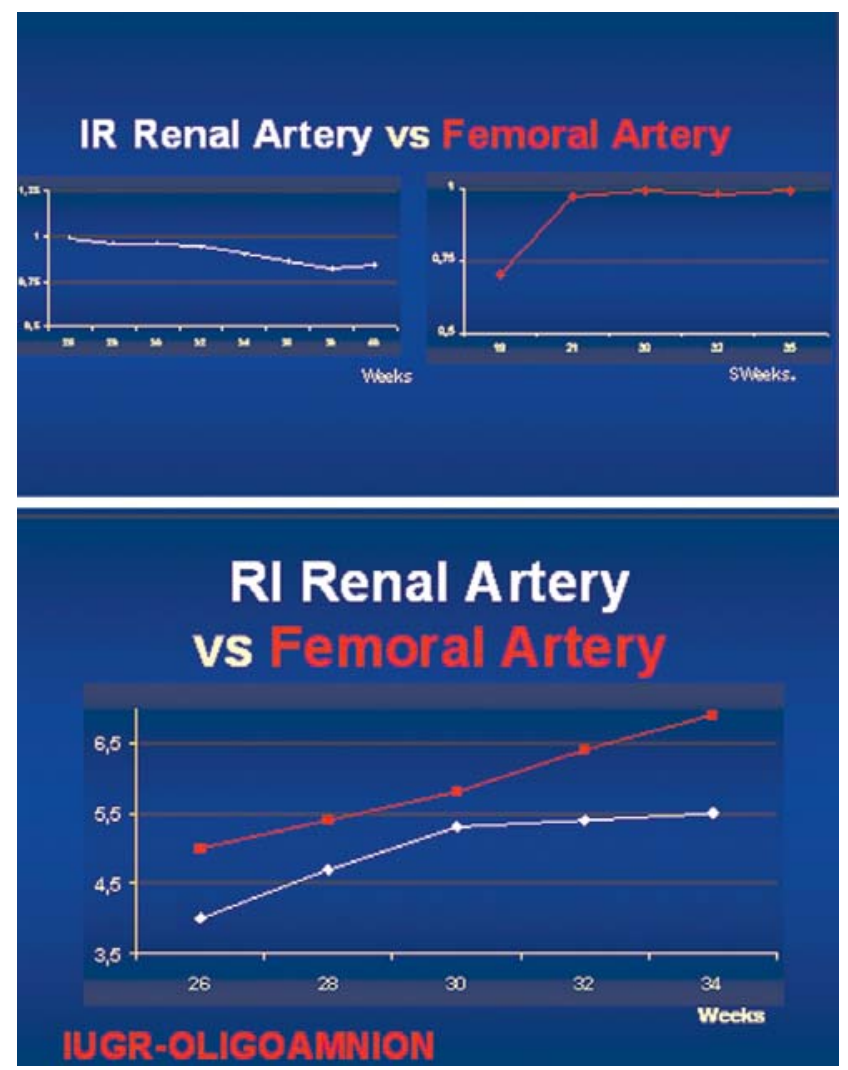

Fig. 15: We observe a negative correlation between renal and femoral flow resistance $(A)$. Such the renal resistance decreased as femoral resistance increased, particularly in situations of chronical fetal distress (B) 


\section{CONCLUSIONS}

The present study has mainly addressed hemodynamic patterns of fetal kidneys, and a number of major conclusions are summarized as follows:

- There is a significant relationship between restrictive RI values and the occurrence of IUGR associated with oligohydramnion.

- There is a positive correlation between RI values and the occurrence of renal pathologies displaying strong dilation.

- There is a positive correlation also between RI values and the co-occurrence of cortico-medullar dysplasia and oligohydramnion.

- Hemodynamic profiles from the renal artery show a more restrictive patterns than those from abdominal vessels, particularly the spleen artery and, to a minor extent, the superior mesenteric, gastric and hepatic arteries.

- The RI decreases throughout gestation in cases of gestational diabetes combined with fetal macrosomy.

- No significant correlation was observed between renal hemodynamics and the appearance of three particular pathologies:

- IUGR in association with euamnion

- Light obstructive flows

- Multicistic dysplasia with euamnion

The monitoring of the hemodynamic patterns emerging from the vessel complex studied here, has allowed an insight into the mechanism of vascular centralization in response to perturbing conditions, this mechanism should be regarded as a kind of functional and nutritional auto-regulative reaction of the visceral set involved.

Fetal kidneys are organs of prime importance in the hemodynamic function of the fetus, and show strong responses to stress in order that:

- Interstitial and glomerullar pressures are maintained within physiological levels, and

- Water losses are minimized for preserving the plasmatic volume and guaranteeing heart outputs.

Hemodynamic patterns resulting from stress consist of the maintenance of a physiological provision with blood for visceral vessels, at the expense of important vascular restrictions focusing upon fetal extremities.

Therefore, fetal extremities are the genuine blood store of the fetus in response to stress of different intensities (physiological acrocianosis in newborns), perhaps the happy fetus has cold feet but a warm heart. ${ }^{11}$

\section{REFERENCES}

1. Peeters LLH, Sheldon RE, Jones MD, Makowsky EL, Meschia G. Blood flow to fetal organs as a function of arterial oxygen content. Am J Obstet Gynecol 1979;135:637-46.

2. Manri G, Moise KJ, Deter RL, Kirshan B, Carpenter RJ, Huhta JC. Doppler assesment of the pulsatility index in the cerebral circulation of the human fetus. Am J Obstet Gynecol 1989;160:698-703.

3. Van der Wiingaard JAGW, Groenenberg IAL, Wladimiroff JW, Hop WCJ. Cerebral Doppler ultrasound in the human fetus. Br J Obstet Gynaecol 1989;96:845-49.

4. Vyas S, Nicolaides KH, Campbell S. Renal artery flow-velocity waveform in normal and hypoxemic fetuses. Am J Obstet Gynecol 1989;161:168-72.

5. Hecher K, Spernol R, Szalay S. Doppler blood velocity waveforms in the fetal renal artery. Arch Gynecol Obstet 1989; 246:133-37.

6. Troyano JM. Fetal hemodynamic profile. Infrequent vascular evaluation. 9th World Congress of Ultrasound in Obstetrics and Gynecology. Buenos Aires 1999.

7. Troyano JM, de la Fuente P. Prenatal aspects about fetal kidney physiopathology and its intra and extrauterine management. Ed. University Hospital of the Canaries 1994.

8. Abuhamad AZ, Mari G, Evans M. Splenic artery flow velocity waveforms in appropiate and small-for-gestational fetuses. Am J Obstet Gynecol 1995;172:820-25.

9. Mari G. Arterial blood flow velocity waveforms of the pelvis and lower extremities in normal and growth retarded fetuses. Am J Obstet Gynecol. 1991;165:143-51.

10. Mercé LT. Velocimetría Doppler durante la gestación tardía. EN: Ecografía Doppler en Obstetricia y Ginecología. Ed. Interamericana-McGraw-Hill 1993;1:200-85.

11. Troyano JM, Clavijo M, Marco OY et al. Fetal hemodynaic profile in uncommon vesels. J Ulr Rev Obstet Gynecol 2003, (3):170-77. 